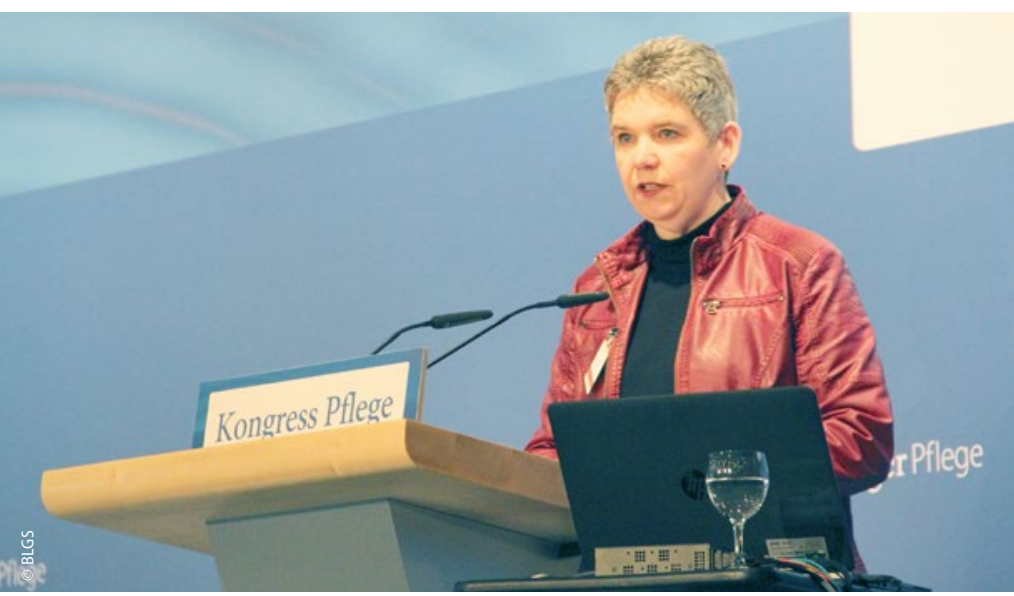

Christine Vogler

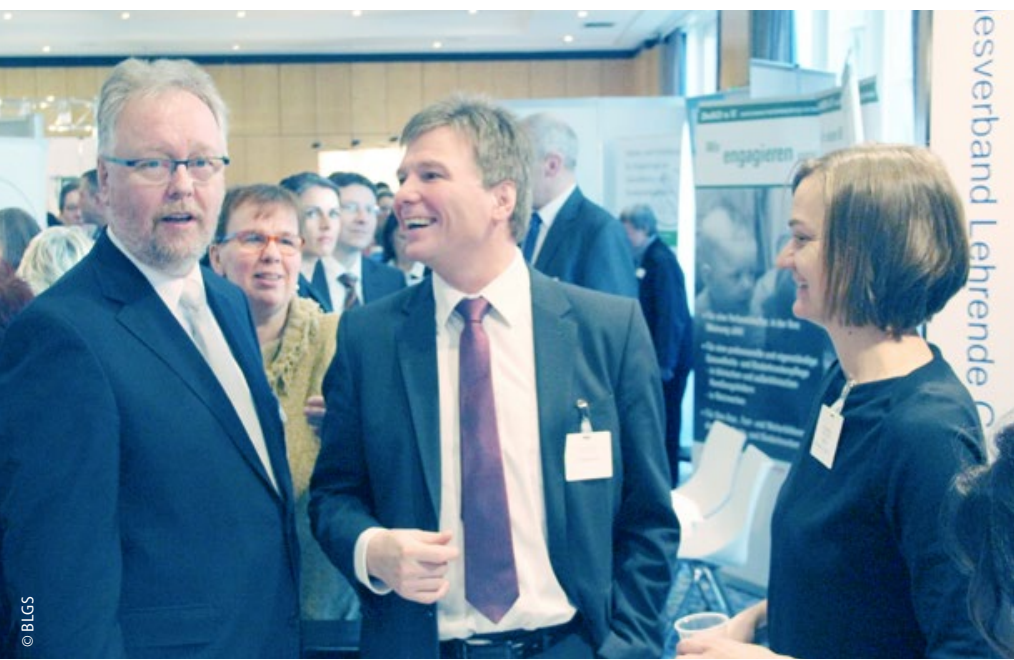

v.I. Michael Breuckmann, Ute Stahl, Uwe Beloch, Angelika Unger

Akademisierte Pflegekräfte

\section{Entwicklung durch Bildung}

Durch die Reform des Pflegeberufsgesetzes hat die Diskussion um die hochschulische Bildung in der Pflege noch einmal an Bedeutung gewonnen. Prof. Dr. Ingrid Darmann-Finck, Professorin für Pflegewissenschaft am IPP der Universität Bremen, hat diesbezüglich die Frage gestellt: Befördert Akademisierung die Entfernung der Mitarbeiter vom Patienten?

Ihrer Studie zufolge sind 48\% der befragten Bachelor-Absolventen in der direkten Pflege tätig. Laut einer Studie des Deutschen Krankenhausinstituts (DKI) sind jedoch 64\% der akademisch qualifizierten Pflegekräfte in Leitungsfunktionen beschäftigt. Entwicklungserfordernisse sind für die Darmann-Finck unter anderem die Definition realistischer Aufgaben und die Schaffung von darauf abgestimmten Arbeitsplätzen. Die Qualifikationen der hochschulisch ausgebildeten Pflegefachpersonen sollten abgefragt und entsprechend entlohnt werden. Außerdem seien Mentoringprogramme erforderlich. Ein Projekt zur Integration akademisierter Pflegekräfte an der Universitätsklinik Regensburg wurde von Kirstin Fragemann vorgestellt. Dr. Doris Arnold und Dr. Alexandra Feiks von der Hochschule in Ludwigshafen referierten über

\section{Kongress Pflege 2016 Von Pflegeberufsgesetz bis Praxisbildung}

Auch in diesem Jahr war der BLGS wieder gut vertreten auf dem Kongress Pflege 2016 in Berlin. Zahlreiche Mitglieder sind angereist, denn neben der Mitgliederversammlung wurde ein interessantes und vielseitiges Programm angeboten. Zwei Programmteile mit insgesamt sieben Vorträgen waren vom BLGS gestaltet worden.

Das Pflegeberufsgesetz bewegt zurzeit alle in der Pflegebildung Beschäftigten. Die Redner Michael Breuckmann, Carsten Drude und Prof. Dr. Wolfgang Heffels sprechen über die wichtigsten Veränderungen des neuen Berufsgesetzes aus den unterschiedlichen Perspektiven Schule und Hochschule. In dem Vortrag „Praxisbildung zwischen Planung und Zufall", der von Christine Vogler moderiert wurde, formulieren Prof. Marlies Beckmann und Ute Müller-Hesselbach die Bedeutung von Pflegediagnosen und Pflegediagnostik in der Ausbildung, sowie die Stolpersteine in der Umsetzung. Ihr Fazit: Die deutsche Pflegepraxis hat großen Nachholbedarf. Sehr spannend war auch der Beitrag von Anne Thiele, die für ihre Masterarbeit das Thema „Belastungen Auszubildender in der Praxis" untersucht hat. Die Ergebnisse zeigen, dass die Unsicherheit der Auszubildenden vor jedem neuen Praxiseinsatz ein sehr zentrales Thema ist. Hieraus zieht Thiele das Resümee, dass der Vorbereitung auf Praxiseinsätze eine besondere Bedeutung beigemessen werden muss. Interessante Perspektiven für die Praxisanleitung zeigt Dr. German Quernheim auf. Diese kann nicht nur den Auszubildenden zugutekommen, sondern auch geschickt als Marketingkriterium genutzt werden. Abschließend beleuchten Prof. Dr. Anke Fesenfeld und Julia Schumacher die Praxisbildung in akademischen Strukturen. Neben dem fachlichen Austausch bot die gelungene Kongressparty am Freitagabend dann wieder Gelegenheit zum Austausch und Kennenlernen.

Monika Dunkmann

das Bildungskonzept und Modellprojekt zur Advanced Nursing Practice, im Rahmen des Gesamtprojektes Entwicklung durch Bildung. Durch akademische Bildung soll das Projekt konkrete Berufsperspektiven für Pflegefachpersonal schaffen. Zudem ist die Sicherung der pflegerischen und gesundheitlichen Versorgung der Westpfalz beabsichtigt. Abschließend sprach Helmut Schiffer, Pflegedirektor der Universitätsklinik Freiburg über den Einsatz akademisierter Pflegekräfte aus Sicht des Managements. Nach dem Motto: Wir überwinden Grenzen in der medizinischen Behandlung, in der Forschung und Lehre und in der Pflege und Personalentwicklung zeigte Fischer die Möglichkeiten von Arbeitsfeldern akademisierter Pflegekräfte, beschrieb die Aktivitäten des eigenen Unternehmens zur Personalförderung und zeigte zukünftige Wege der hochschulischen Bildung in Freiburg auf. Fakt ist, dass der erfolgreiche Einsatz von hochschulisch gebildeten Pflegekräften bereits heute Realität ist. Dies gilt es weiter auszubauen und in den Regelbetrieb zu überführen. 